\title{
An Investigation of the Accuracy of Finite-Volume Radial Domain Truncation Technique
}

\author{
Krishnaswamy Sankaran, Christophe Fumeaux and Rüdiger Vahldieck \\ Laboratory for Electromagnetic Fields and Microwave Electronics - IFH \\ ETH Zurich \\ Zurich, CH-8092, Switzerland, Email: ksankaran@ieee.org \\ Telephone: +414463266 71, Fax: +41446321198
}

\begin{abstract}
The accuracy and performance of the radial domain truncation technique is presented in the framework of finite-volume time-domain method. In the present approach all the electromagnetic field quantities are co-located in both space and time and the performance is evaluated using unstructured grid. The influence of the radius of curvature of the absorber is investigated using a waveguide and a hornantenna as practical examples. Numerical reflection errors are computed using a reference solutions and the convergence of the results is studied for increasing radius of curvature of the absorber. Low-level effects on the antenna radiation patterns further illustrates the convergence of the technique.
\end{abstract}

Index Terms-Finite-Volume Time-Domain-FVTD, Radial Domain Truncation, Perfectly Matched Layer-PML, Absorbing Boundary Condition-ABC, Computational Electromagnetics-CEM.

\section{INTRODUCTION}

The Finite-Volume Time-Domain (FVTD) method belongs to the class of conformal time-domain method which has the advantage of employing unstructured spatial discretization. This helps in modeling complex geometries without stair-casing error which is typical to classical Finite-Difference Time-Domain (FDTD) method. In addition, the FVTD method allows an explicit timediscretization which avoids computational intensive matrix inversions typical to implicit time-domain methods. In the present approach, the electric and magnetic field values are both spatially and temporally co-located. An investigation of radial domain truncation technique in the framework of FVTD method is presented and the influence of the radius of curvature of the absorber is examined. The radial absorber is modeled as an unsplit perfectly matched layer (PML) [1], [2], [3] on unstructured finite-volume grid. This model satisfies Maxwell equations both inside and outside the absorber region without any unphysical field-splitting as in the case of Berenger's split-PMLs [4], [5]. The radial perfectly matched layer technique for the FVTD method [6] has the advantage of simplified update equations, reduced computational domain and geometrymatched modeling for cylindrical and elongated structures. However, it is observed that the radius of curvature of radial absorber has a noticable influence on its perfor- mance. Hence, this paper explores the accuracy limitations of the radial absorber based on its radius of curvature and examines the usability of the model for studying practical problems. The structure of the paper is as follows. The mathematical preliminaries of the FVTD method are briefly introduced in Sec. II and the theory of radial absorber is discussed in Sec. III. The influence of the radius of curvature is investigated in Sec. IV by employing radial absorbers of different radii to truncate a rectangular waveguide. The numerical experiment setup presented in Sec. V consists of a horn-antenna example. Numerical reflection and its effect on the computed radiation pattern are analyzed for different radii of curvature of the absorber. Sec. VI concludes the paper with a summary of the findings and discussion of the results.

\section{FVTD APPROACH}

The basic terminology used in the FVTD method is briefly reviewed in this section in order to make the paper self-sufficient. Interested readers are refered to [7], [8], [9]. One of the main goal in computational electromagnetics is to model the continuous Maxwell system using discrete space and time samples. In the context of the FVTD method each discrete space sample $\Omega_{i}$ is called controlvolume or cell. The time sampling is characterized by the discrete temporal increment $\Delta t$ which is used to update the solution in the time-marching algorithm. Depending on the method of storing and updating the field quantities, different variations in the FVTD algorithm are possible. In order to keep the analysis simple, a 2D transverse electric $(T E)$ model with the electric field along the $z$-axis $\left(E_{z}\right)$ and the magnetic field in the $x y$-plane $\left(H_{x}\right.$ and $\left.H_{y}\right)$ is considered. The two Maxwell curl equations integrated over each $i$-th polyhedral cell with control-volume $\left|V_{i}\right|$ bounded by $f$ faces is transformed into a discrete FVTD system with the help of divergence theorem as follows [9],

$$
\alpha \partial_{t} \boldsymbol{U}=-\frac{1}{\left|V_{i}\right|} \sum_{k=1}^{f}\left(\mathcal{F}_{\boldsymbol{U}_{k}} \cdot \boldsymbol{n}_{k}\left|S_{k}\right|\right)-\alpha \partial_{t} \boldsymbol{Q}
$$

where $\boldsymbol{U}=\left[H_{x}, H_{y}, E_{z}\right]^{T}$ denotes the EM field-vector with the superscript ' $\mathrm{T}$ ' representing matrix transpose. 
Each $k$-th face has an surface area of $\left|S_{k}\right|$ and a unit outward-normal $\boldsymbol{n}_{k}$. The term $\alpha$ represents the material parameters namely permeability $\left(\mu_{i}\right)$ and permittivity $\left(\varepsilon_{i}\right)$ for the magnetic and electric field update-equations, respectively. The vector $\boldsymbol{Q}=\left[M_{x}, M_{y}, P_{z} / \varepsilon_{0}\right]^{T}$ represents the components of magnetization $\boldsymbol{M}$ and polarization $\boldsymbol{P}$ fields inside the medium. The factor ' $\mathcal{F}_{\boldsymbol{U}_{k}} \cdot \boldsymbol{n}_{k}$ ' is called the FVTD $f u x$-function which contributes to the information exchange between adjacent cells. In the present work, the time update is done using a second order accurate LaxWendroff scheme [9] and the spatial discretization involves unstructured triangular meshing of the computational domain.

\section{THEORY OF RADIAL ABSORBER}

The fundamental idea in the theory of radial absorber is to transform the locally uniaxial absorber material properties [2] to globally radial material properties. In order to do this, the Maxwell system is initially represented in the cylindrical coordinate system and later, a transformation is introduced to represent uniquely the radial system using only the cartesian components [6]. This transformation is in fact, a rotation by an angle $\varphi$ in 2D about the $z$-axis which affects only the components in $x y$-plane. The final system of equations representing the radial absorber model is written using the flux-based FVTD update equations as follows,

$$
\begin{aligned}
\partial_{t} H_{x}= & \frac{-\sum_{k=1}^{f}\left(\mathcal{F}_{H_{x k}} \cdot \boldsymbol{n}_{k}\left|S_{k}\right|\right)}{\mu_{0}\left|V_{i}\right|} \\
& -K_{x_{r}} \cos \varphi-\zeta_{x_{r}} H_{x} \sin ^{2} \varphi \\
& +\zeta_{x_{r}} H_{y} \cos \varphi \sin \varphi \\
\partial_{t} H_{y}= & \frac{-\sum_{k=1}^{f}\left(\mathcal{F}_{H_{y k}} \cdot \boldsymbol{n}_{k}\left|S_{k}\right|\right)}{\mu_{0}\left|V_{i}\right|} \\
& -K_{x_{r}} \sin \varphi-\zeta_{x_{r}} H_{y} \cos ^{2} \varphi \\
& +\zeta_{x_{r}} H_{x} \cos \varphi \sin \varphi \\
\partial_{t} E_{z}= & \frac{-\sum_{k=1}^{f}\left(\mathcal{F}_{E_{z k}} \cdot \boldsymbol{n}_{k}\left|S_{k}\right|\right)}{\varepsilon_{0}\left|V_{i}\right|}-\zeta_{x_{r}} E_{z} \\
\partial_{t} K_{x_{r}}= & \zeta_{x_{r}} \sin \varphi \frac{\sum_{k=1}^{f}\left(\mathcal{F}_{H_{y k}} \cdot \boldsymbol{n}_{k}\left|S_{k}\right|\right)}{\mu_{0}\left|V_{i}\right|} \\
& +\zeta_{x_{r}} \cos \varphi \frac{\sum_{k=1}^{f}\left(\mathcal{F}_{H_{x k}} \cdot \boldsymbol{n}_{k}\left|S_{k}\right|\right)}{\mu_{0}\left|V_{i}\right|}
\end{aligned}
$$

The term $\zeta_{x_{r}}$ corresponds to the loss in the radial absorber which is uniaxial along the $x_{r}$-direction. The scalar field $K_{x_{r}}$ corresponds to the magnetic counterpart of polarization current as defined in [2] and [10]. It should be noticed that the flux terms computed for (2) and (3) can be reused in (5) which helps in reducing computational effort. When the rotation angle $\varphi$ is equal to 0 or 90 degree, the resulting system corresponds to uniaxial absorber in $x$ and $y$ directions, respectively. Furthermore, these uniaxial absorber models are the limiting case of the radial absorber when the radius of curvature $\left(r_{\mathrm{C}}\right)$ approaches infinity. In other words, when varying $r_{\mathrm{C}}$ from very small value to higher values, the radial absorber model approaches the uniaxial absorber model. This concept is graphically illustrated in Fig. 1.
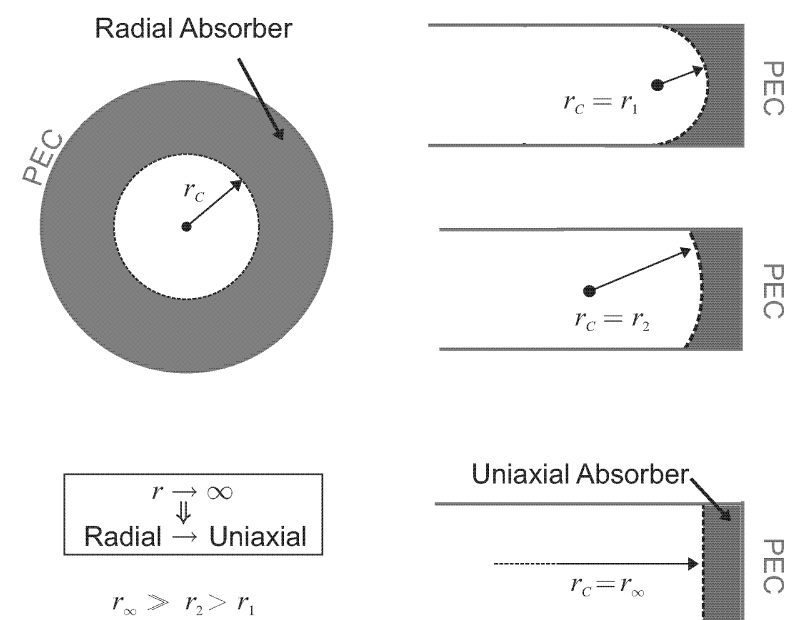

Fig. 1. Left: Cylindrical domain truncation. Right: Waveguide truncation emphasizing that the uniaxial absorber is a limiting case of radial absorber with infinite radius of curvature.

\section{INFLUENCE OF THE RADIUS OF CURVATURE}

As a practical illustration of the concept introduced in the last section, absorbers with different radius of curvature as shown in the right-hand side of Fig. 1 are used to truncated a rectangular waveguide. The goal of this approach is to study the influence of the radius of curvature $r_{\mathrm{C}}$ and compare it against theoretically expected values. Furthermore, the results are compared with those of first-order Silver-Müller Absorbing Boundary Condition (SM-ABC) which is the most commonly used ABC in the FVTD method. The results of this study are presented in Fig. 2. The loss term $\zeta_{x_{r}}$ is chosen with a quadratic profile such that at normal incidence the theoretical reflection coefficient is $-70 \mathrm{~dB}$ [4]. By using the plane wave decomposition model of a waveguide mode, changing the frequency of the incident mode is equivalent to changing the angle of incidence with respect to the free spaceabsorber interface. At the cut-off frequency $f_{c}$ the angle of incidence corresponds to 90 degree and as the frequency of the incident mode increases the angle of incidence asymptotically reaches 0 degree. Consequently, for each frequency (angle of incidence), the theoretical return-loss $S_{11}$ is uniquely defined for a truncated waveguide and it is shown as dashed line in Fig. 2. Clearly the performance of the SM-ABC is poor in comparison with even the 


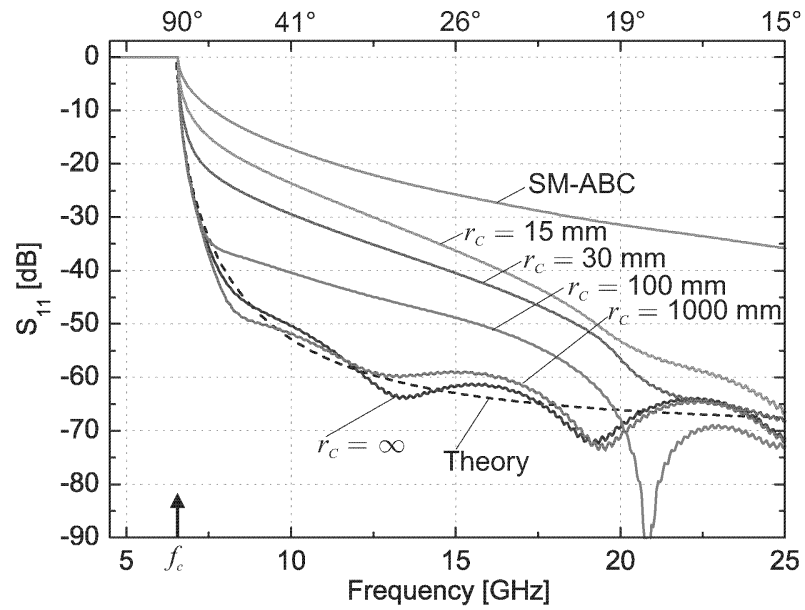

Fig. 2. Waveguide truncation showing the convergence of the radial absorber with increasing radius of curvature $r_{\mathrm{C}}$ towards uniaxial absorber model. The performance of first order SM-ABC is also shown as a comparison. The angle of incidence corresponding to each frequency is also indicated on the upper horizontal scale. The theoretical results at each angle is computed such that a PML reflection coefficient $-70 \mathrm{~dB}$ is achieved at normal incidence.

radial absorber with the smallest radius. It can be noticed that all the radial aborber models converge towards the theoretically expected value of $-70 \mathrm{~dB}$ as the frequency of the incident mode increases. In particular, the rate of convergence is quite fast in the case of radial absorber with large radii. Furthermore, there can be another interpretation on the convergence of the results: As the radius of curvature increases, the computed reflection coefficient of the radial absorber converge towards the theoretical curve of the uniaxial absorber $\left(r_{\mathrm{C}}=\infty\right)$.

\section{NUMERICAL EXPERIMENT: HORN ANTENNA}

The performance of the radial absorber discussed in the previous section is investigated for an antenna radiation problem using radial absorbers with different radii as shown in the Fig. 3. For the sake of clarity the finite thickness of the radial absorber is not depicted in the illustration. For all simulations the thickness of the radial absorber is chosen as $d_{a b s}=1 \lambda_{\min }$ where $\lambda_{\min }$ corresponds to wavelength of the maximum frequency of interest $f_{\max }$. This approximately corresponds to 15 layers of unstructured triangular cells. The goal of this study is to examine the influence of $r_{\mathrm{C}}$ on the domain truncation accuracy. The experimental setup consists of a small 2D horn-antenna with infinite extension along the $z$-axis. The length of the flared section along the $x$-axis is $31.3 \mathrm{~mm}$ and the aperture size is $44.30 \mathrm{~mm}$. The horn-antenna is fed by a waveguide with width $22.84 \mathrm{~mm}$ and operated in its fundamental $T E_{10}$ mode. The source of excitation is a sine-modulated Gaussian pulse with bandwidth streching from 8-12.5 GHz. The near-field-to-far-field (NF-FF) Huy-

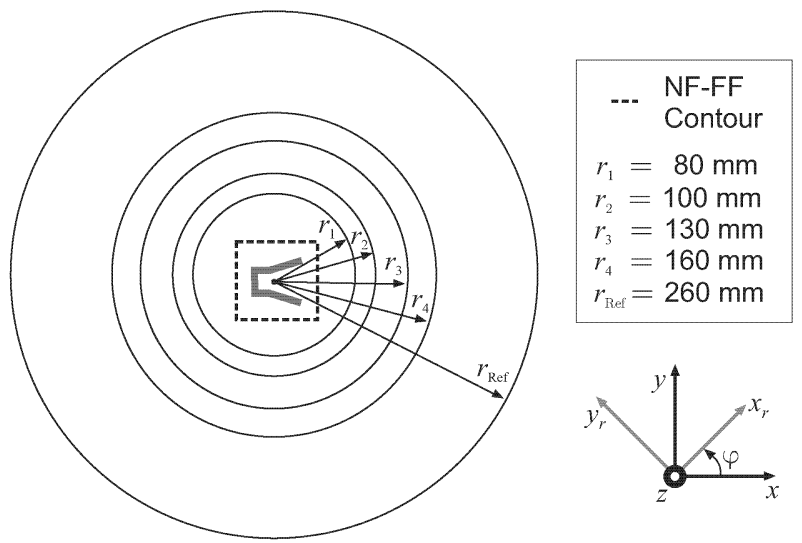

Fig. 3. Computational Domain consisting of 2D horn-antenna with different $r_{\mathrm{C}}$. The NF-FF contour used to compute antenna radiation pattern is shown.

gens contour used to compute the antenna radiation pattern is also shown in the Fig. 3. Snapshots of the reflected electric field from the radial domain truncation are shown for different radius of curvature in Fig. 4. The reflected field patterns are obtained by subtracting the corresponding field patterns of the radially truncated model from those of a large reference model where simulation is stopped before the reflections reach the considered domain. The illustrated snapshots are taken after the incident pulse left the considered computational domain. As noticed from the Fig. 4, there is a focusing effect in the reflected field pattern which originates due to the concave geometry of the radial truncation. However, this focusing effect decreases as the radius of curvatures increases. Furthermore, there is a visible decrease in the reflected field as the radius of curvature increases.

The radiation pattern of the horn-antenna excited by fundamental $\mathrm{TE}_{10}$ mode is computed. The numerical results were validated using measurement data for the mainlobe. However, due to the limitations in the measurements chambers the low-level back radiations of the horn-antenna are not accurately resolved. Hence, the numerical results from the larger model are used as reference solution. The results of this analysis are shown in Fig. 5. The influence of the radius of curvature is only noticable in the lowlevel side-lobe, as expected because the reflections from the radial absorber are well below $-40 \mathrm{~dB}$. The radial absorber with the smallest radius $r_{\mathrm{C}}=80 \mathrm{~mm}$ exhibits the highest deviation from the reference model. As the radius increases the results converge as expected.

\section{CONCLUSION}

From various experiments and results presented in the previous section, the influence of the radius of curvature of the absorber was investigated. It was observed that the radius of curvature has a noticable impact on the accuracy 

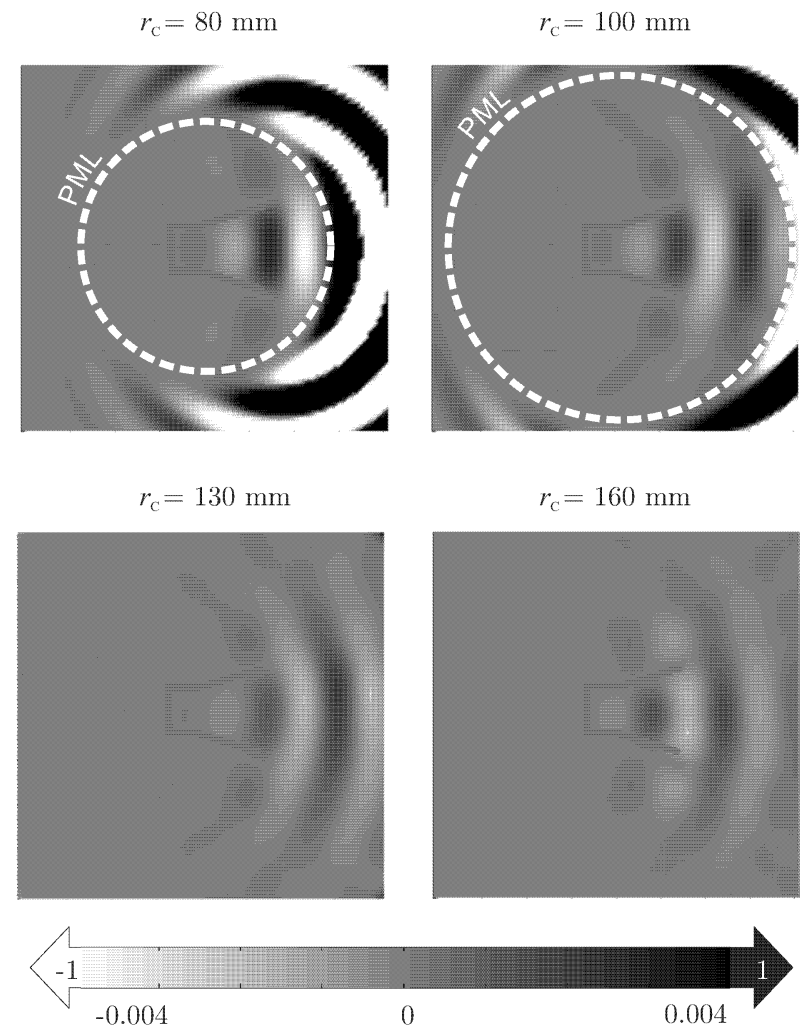

Fig. 4. Reflections from radial absorber of different radii used to truncate the domain consisting of radiating horn-antenna excited by first-order $\mathrm{TE}_{10}$ mode. The free-space - absorber interface is depicted in the case of $r_{\mathrm{C}}=80 \mathrm{~mm}$ and $r_{\mathrm{C}}=100 \mathrm{~mm}$. In the other cases the free-space radial absorber interface are further away and therefore, not shown in the two bottom plots. The area outside the dashed circles corresponds to the radial PML and shows a large difference with respect to the reference because of the absorption in the radial PML.

of the absorber. In certain cases, a focusing effect was observed. This focusing effect is a trade-off for cornerreflections from standard rectangular PML. It is should stressed that, the results presented in this paper are only a worst-case scenario and further investigations on the performance of radial absorbers are subject of future research. For most practical applications like antenna pattern computation, the observed reflection errors do not affect significantly the computed results and hence, it is possible to place the radial absorber very close to the radiating source. Consequently, the radial absorber can aid in reducing the total computational domain required maintaining certain level of accuracy. Furthermore, the radial absorber can be used at its full advantage for applications involving very low-level coupling or scattering phenomena.

\section{ACKNOWLEDGEMENT}

This work is supported by the ETH Research Grant TH38/04-1.

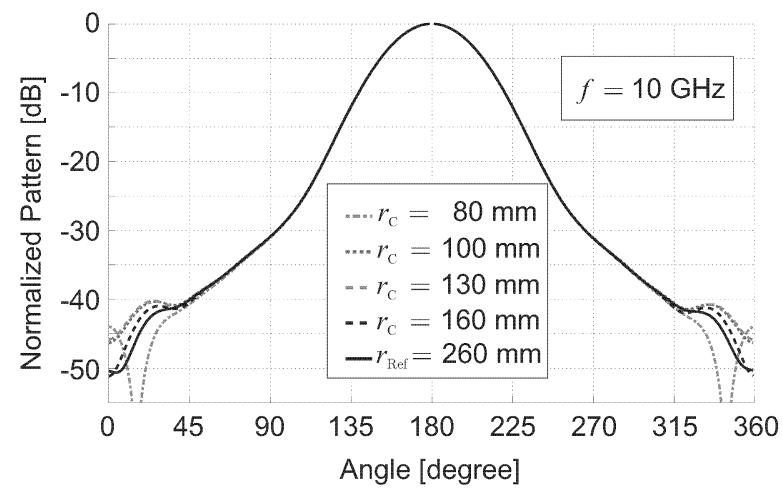

Fig. 5. Horn-antenna radiation pattern at $10 \mathrm{GHz}$ using radial absorber of different radii. The results shown nearly same results for the main-lobe of the antenna. Differences are observable in the side-lobes which are well below $-40 \mathrm{~dB}$. Convergence in the result is noticed as $r_{\mathrm{C}}$ increases.

\section{REFERENCES}

[1] S. Gedney, "An anisotropic perfectly matched layer-absorbing medium for the truncation of FDTD lattices," IEEE Transactions on Antennas and Propagation, vol. 44, no. 12, pp. 1630-1639, December 1996.

[2] R. W. Ziolkowski, "Time-derivative Lorentz material model-based absorbing boundary condition," IEEE Transactions of Antennas and Propagation, vol. 45, no. 10, pp. 1530-1535, October 1997.

[3] L. Zhao and A. Cangellaris, "GT-PML: Generalized theory of perfectly matched layers and its application to the reflectionless truncation of finite-difference time-domain grids," IEEE Transactions on Microwave Theory and Techniques, vol. 44, no. 12, pp. 2555-2563, December 1996.

[4] J.-P. Bérenger, "A perfectly matched layer for the absorption of electromagnetic waves," Journal of Computational Physics, vol. 114, no. 2, pp. 185-200, 1994.

[5] _ , "Three-dimensional perfectly matched layer for the absorption of electromagnetic waves," Journal of Computational Physics, vol. 127, no. 2, pp. 363-379, September 1996.

[6] K. Sankaran, C. Fumeaux, and R. Vahldieck, "Uniaxial and radial anisotropy models for finite-volume maxwellian absorber," IEEE Transactions on Microwave Theory and Technique, vol. 54, no. 12, December 2006.

[7] N. K. Madsen and R. W. Ziolkowski, "A three-dimensional modified finite volume technique for Maxwell's equations," Electromagnetics, vol. 10, pp. 147-161, 1990.

[8] V. Shankar, A. H. Mohammadian, and W. F. Hall, "A time-domain, finite-volume treatment for the Maxwell equations," Electromagnetics, vol. 10, pp. 127-145, 1990.

[9] P. Bonnet, X. Ferrieres, B. Michielsen, P. Klotz, and J. Roumiguiéres, Time Domain Electromagnetics. S. M. Rao, Ed., Academic Press, 1997, ch. 9, pp. 307-367.

[10] K. Sankaran, C. Fumeaux, and R. Vahldieck, "Finite-volume Maxwellian absorber on unstructured grid," 2006 IEEE MTT-S International Microwave Symposium Digest, pp. 169-172, June 2006. 